Estudios de la Paz y el Conflicto

Revista Latinoamericana

IUDPAS-UNAH

Volumen 1, Número 1, pp. 39-58

ISSN-e: 2707-8922 / ISSN: 2707-8914

DOI: $10.5377 /$ rlpc.v1i1.9515

Enero-junio 2020

\title{
ANÁLISIS NORMATIVO DEL BIENESTAR ANIMAL EN ESPAÑA Y LATINOAMÉRICA: ESPECIAL REFERENCIA A LA LABOR DE LOS PERROS EN LA CONSTRUCCIÓN DE PAZ
}

\author{
REGULATORY FRAMEWORK OF ANIMAL WELFARE IN LATIN AMERICA AND \\ SPAIN: SPECIAL REFERENCE TO THE DOGS CONTRIBUTION FOR BUILDING \\ PEACE
}

\author{
Marta Méndez Juez \\ Universidad de Burgos \\ mmjuez@ubu.es \\ Juan Luis de Castellví Guimerá \\ Parlamento Europeo \\ jlcastellvi@gmail.com
}

Cómo citar / citation

Méndez, M. y de Castellví, J. (2020). “Análisis normativo del bienestar animal en España y Latinoamerica: especial referencia a la labor de los perros en la construcción de paz", Estudios de la Paz y el Conflicto, Revista Latinoamericana, Volumen 1, Número 1, 39-58. DOI: $10.5377 /$ rlpc.v1i1.9515

\begin{abstract}
Resumen
La protección del bienestar animal es hoy día una preocupación universal. Sin embargo, su garantía sigue siendo una laguna que aún no ha cubierto el derecho internacional. Desde la dimensión ambiental del modelo de paz transformadora, el desarrollo del Buen Vivir es fundamental para entender nuestras relaciones sociales y nuestras interacciones con el resto de seres vivos. Por ello, trataremos de entender qué papel desempeñan los animales y, en concreto, los perros en la cultura de paz. Veremos cómo su labor puede resultar de gran trascendencia en ámbitos de actuación pública esenciales en cualquier modelo de sociedad, como son la sanidad, la salud o las emergencias. Posteriormente, destacaremos algunas experiencias latinoamericanas que ilustran ese cambio en la concepción del bienestar animal y su aportación a las sociedades actuales. Estas experiencias llaman al optimismo y que pueden ser propulsoras del cambio social hacia modelos de convivencia que comporten un beneficio común para animales y humanos, en las interacciones que puedan generarse entre ambos. Finalizaremos exponiendo algunas conclusiones y
\end{abstract}


reflexiones generales que inciten a futuros investigadores a trabajar sobre esta misma materia. Queda mucho camino por recorrer, pero ya hemos empezado a andar.

\title{
Palabras clave
}

Paz; Latinoamérica; Perro; Bienestar; Buen Vivir; Ser sintiente; Terapia.

\begin{abstract}
The protection of animal welfare is a universal concern today. However, its guarantee remains a gap that has not yet been covered by international law. From the environmental dimension of the transformative peace model, the development of Good Living is essential to understand our social relations and our interactions with other living beings. Therefore, we will try to understand what role animals and, in particular, dogs play in the culture of peace. We will see how their work can be of great importance in areas of public action essential in any model of society, such as health, health or emergencies. Later, we will highlight some Latin American experiences that illustrate this change in the conception of animal welfare and its contribution to today's societies. These experiences call for optimism and that can be drivers of social change towards convivial models that have a common benefit for animals and humans, in the interactions that can be generated between them. We will conclude by presenting some conclusions and general reflections that encourage future researchers to work on the same subject. There is a long way to go, but we have already begun to walk.
\end{abstract}

\section{Keywords}

Peace; Latin America; Dog; Wellness; "Buen Vivir”; Sentient Beings; Therapy.

\section{INTRODUCCIÓN}

El paso más difícil es salir de nosotros mismos y volver la vista atrás hacia dónde y cómo vivimos (Safina, 2016: 473). Tradicionalmente, hemos entendido a la paz en su concepción negativa, aquella que establece una relación de oposición entre paz y guerra; y un estrecho vínculo entre paz, orden y control. Pero más allá de ser el resultado de eliminar una violencia directa, se trata de construir paz positiva, de palpables contenidos pacíficos que mejoren la calidad de la vida de las personas y atiendan a las necesidades humanas básicas. Avanzando más en esta concepción de paz, las últimas investigaciones confluyen en señalar que la paz sólo se comprende como un constructo social en permanente transformación que se desarrolla cuando logramos cristalizar modelos de vida en nuestras, comunidades, sociedad y sistemas políticos y económicos que facilitan el desarrollo de las potencialidades humanas del conjunto de la población. Es por ello que se afirma que la paz es el camino (y no la meta) que construimos los seres humanos participativamente cuando nos comprometemos al desarrollo del Buen Vivir. A este modelo se le denomina paz transformadora.

Y desde este modelo de paz transformadora, el desarrollo del Buen Vivir o Vivir Bien o Sumak Kawsay es fundamental para entender nuestras relaciones sociales y nuestras interacciones con el resto de seres vivos. El Buen Vivir es el proceso de la vida en plenitud, vivir en armonía con los demás seres y la naturaleza, sobre la base de la unidad, la solidaridad, la empatía y el equilibrio natural. Desde una dimensión ambiental, la construcción de paz supone respetar y cuidar la Madre Tierra porque es el soporte de la vida en el planeta. En 
definitiva, entender el enfoque de paz transformadora en su dimensión ambiental, y potenciarla desde la cultura del Buen Vivir, es un buen punto de partida para explorar las posibilidades de interacción mutua que existen entre los animales y los seres humanos, entre los perros y las personas.

Tal es así que ciertos países latinoamericanos llevan tiempo promoviendo la aprobación definitiva, en la Organización de Naciones Unidas, de una Declaración Universal sobre los Derechos de la Madre Tierra, que incluya específicamente la protección animal. Más allá del debate filosófico y doctrinal que pueda generarse sobre el establecimiento o no del derecho de un animal, se trataría más bien de determinar los límites de la actuación humana en el aprovechamiento de esta especie. De igual modo, así como es preciso limitar el comportamiento humano hacia cualquier práctica que genere dolor, sufrimiento o daño a los animales, sería conveniente analizar y legislar aquellas prácticas que, no siendo nocivas para los animales, comporten un beneficio común para estos y los humanos, en las interacciones que puedan generarse entre ambos. Especialmente, las relaciones que se producen entre perros y humanos, a menudo resultan muy beneficiosas para las personas en ámbitos como la sanidad, la educación o la seguridad, sin causar efectos lesivos para los canes.

Y sobre esto versará el contenido del presente artículo. Primero, plantearemos conceptos esenciales en esta materia, como pueden ser la paz, la seguridad, el bienestar animal, la cultura del Buen Vivir o la etología, a fin de comprender el marco teórico en el que basamos nuestras aportaciones. En segundo lugar, trataremos de entender qué papel desempeñan los animales y, en concreto, los canes en la cultura de paz, desde la dimensión ambiental de la paz transformadora. Veremos cómo su labor puede resultar de gran trascendencia en ámbitos de actuación pública esenciales en cualquier modelo de sociedad, como son la sanidad, la salud o las emergencias. Posteriormente, destacaremos algunas prácticas o ciertos ejemplos latinoamericanos que ilustran ese cambio en la concepción del bienestar animal y su aportación a las sociedades actuales. Finalizaremos exponiendo algunas conclusiones y reflexiones generales que inciten a futuros investigadores a trabajar sobre esta misma materia.

\section{METODOLOGÍA}

El estudio realizado es de carácter exploratorio. La finalidad del mismo es encontrar el marco normativo idóneo para facilitar la actividad prestacional de los perros en sociedad: específicamente, en relación a procesos de construcción de paz.

Aplicando técnicas de análisis normativo, hemos querido comprobar cuál es el marco institucional del que partimos para proponer escenarios futuros de mejora en el sector. Y para eso, hemos comparado el ordenamiento jurídico español con algunos latinoamericanos, al aportar ambos perspectivas diferentes. El análisis realizado nos ha permitido concluir que las experiencias latinoamericanas son un ejemplo muy ilustrativo que demuestra cómo pensar en los animales como seres sintientes, procurar su bienestar y aprovechar su potencial para diferentes tareas de la vida cotidiana, la mejora de la convivencia y la contribución a la construcción de paz.

Estudios de la Paz y el Conflicto, Revista Latinoamericana, IUDPAS-UNAH, Volumen 1, Número 1, enero-junio 2020, pp. 39-58. DOI: 10.5377/rlpc.v1i1.9515 


\section{BIENESTAR ANIMAL, BUEN VIVIR Y PAZ TRANSFORMADORA}

La paz puede ser desde un concepto filosófico hasta un objetivo de trabajo de diversas organizaciones internacionales, pero su impacto en la vida diaria es un hecho tangible, especialmente en el caso de su ausencia. Así:

"El concepto de paz ha evolucionado desde la segunda guerra mundial, pues no es sólo ausencia de conflictos o guerra, sino es un fin, un objetivo imprescindible para ejercer y disfrutar los derechos humanos, en otras palabras, la paz, es sinónimo de promoción y respeto de los derechos fundamentales (...), la paz, valor universal anhelado y necesario en el mundo, no se ha cristalizado y es precaria, debido a las constantes amenazas producto de la violencia, que se da en todos los ámbitos, en los estados, en conflictos armados, en actos terroristas, en los hogares, por el armamentismo y las armas nucleares, por el antagonismo entre los Estados, por las divisiones ideológicas y religiosas, por la injusticia, la represión, la explotación económica y en general por el menosprecio de la dignidad del hombre" (Arango, 2007: 10).

Tradicionalmente, hemos entendido a la paz en su concepción negativa, aquella que establece una relación de oposición entre paz y guerra; y un estrecho vínculo entre paz, orden y control. Pero más allá de ser el resultado de eliminar una violencia directa, se trata de construir paz positiva, de palpables contenidos pacíficos que mejoren la calidad de la vida de las personas y atiendan a las necesidades humanas básicas. Avanzando más en esta concepción de paz, las últimas investigaciones confluyen en señalar que la paz sólo se comprende como un constructo social en permanente transformación que se desarrolla cuando logramos cristalizar modelos de vida en nuestras, comunidades, sociedad y sistemas políticos y económicos que facilitan el desarrollo de las potencialidades humanas del conjunto de la población. Es por ello que se afirma que la paz es el camino (y no la meta) que construimos los seres humanos participativamente cuando nos comprometemos al desarrollo del Buen Vivir. A este modelo se le denomina paz transformadora (Ramos, 2015).

Paralelamente al estudio de la paz, se suele analizar el término de seguridad. Etimológicamente, la palabra "seguridad" proviene del latín sine cura (sin cuidado, sin preocupación), pero esta definición se queda muy escueta por la complejidad y extensión de los asuntos a que debe hacer frente, al aumento de nuestras capacidades analíticas. Tal es así que los seres humanos han distinguido y diferenciado distintos ámbitos de seguridad y una gran variedad de objetos de estudio tratados por la disciplina (seguridad nacional, la seguridad en el trabajo, la seguridad social, la seguridad ciudadana, ecológica, económica, alimentaria, entre otras). La Asamblea General de Naciones Unidas (ONU, 2005) definió la seguridad desde tres principios esenciales: "libertad para vivir sin temor", "libertad para vivir sin miseria" y "libertad para vivir con dignidad".

Y hablar de paz y seguridad es también hablar del concepto de seguridad humana, acuñado por la Organización de Naciones Unidas (PNUD, 1994) y del que destacan cuatro características básicas de dicha seguridad: universalidad, interdependencia de sus componentes, centrada en las personas y mejor garantizada mediante la prevención temprana. Además, el Informe establecía siete ámbitos relacionados: seguridad económica, alimentaria, de la salud, del medio ambiente, personal, comunitaria y política. Nos interesa, precisamente, hacer hincapié en el hecho de que la seguridad humana también implica seguridad ambiental, reconociendo y garantizando el medio en que vivimos y a cuantos seres habitamos en él.

Podemos ver cómo hoy, ese medio en que vivimos, la naturaleza, está incluso reconocida como sujeto de derecho por algunas constituciones latinoamericanas, como es el caso de la

Estudios de la Paz y el Conflicto, Revista Latinoamericana, IUDPAS-UNAH, Volumen 1, Número 1, enero-junio 2020, pp. 39-58. DOI: 10.5377/rlpc.v1i1.9515 
Constitución ecuatoriana ${ }^{1}$. Sin embargo, a otros seres que habitan en él, a los animales aún no se les ha otorgado el estatuto jurídico que merecen a pesar de que se ha demostrado que tienen la capacidad de expresar sus sentimientos y sentir empatía.

Y es aquí donde surge el término de bienestar animal ${ }^{2}$. Sin duda, se trata de es un tema multidimensional, que necesita un enfoque integrativo de varias disciplinas diferentes como la fisiología, la ciencia veterinaria, la etología, la paleontología o la psicología social. Una de las esferas de conocimiento más relevantes en el análisis del bienestar animal es la etología. La etología (literalmente, ciencia de la costumbre), describe y analiza el comportamiento de los animales en su ambiente natural y explica por qué lo hacen, ayuda a entender sus necesidades y a conseguir una convivencia ordenada y equilibrada con personas, y que se relacione con normalidad con el entorno y los estímulos que le rodean. Superando la creencia de que nunca podríamos saber cómo se sienten los animales, sino sólo cómo se comportan, algunos etólogos y psicólogos cognitivos (Dawkins, 1993) han realizado estudios de percepción, llegando a la conclusión de que la especialización de los grupos celulares en los animales les permite tener un sistema nervioso altamente evolucionado en la actualidad (Yánez, 2015).

Dentro del proceso evolutivo del ser humano y su sociedad, la interacción con los animales ha ido cambiando, iniciándose un proceso de domesticación de algunas especies animales, especialmente de perros. Es así como actualmente su colaboración con ser humano no es únicamente de tipo productivo, sino que encontramos animales de compañía, de terapia o de asistencia (entre otros), que son educados y cuidados para la convivencia con los seres humanos (Hernández y Fuentes, 2018). Pero, ¿cómo se asegura su bienestar? ¿Tienen derechos al igual que nosotros los humanos como seres vivos que somos?

En la actualidad, la ausencia de un instrumento marco sobre la protección del bienestar animal es una laguna en el derecho internacional. Por lo tanto, se hace necesario que la comunidad internacional converja en la concepción universal de la protección del bienestar animal en el derecho internacional (Yánez, 2015). De hecho, la Declaración A/66/750 (ONU, 2012), ya abordó la problemática de la protección del bienestar animal y se plantearon en ella los objetivos de consumo y producción sostenibles, como metas a lograr entre 2012-2020.

El Derecho Internacional no es la panacea para resolver todos los problemas en el mundo, como tampoco lo es el legislar taxativamente sobre todas las materias. Sin embargo, este puede evidenciar una preocupación compartida y considerar la protección del bienestar animal como un objetivo común por todos los Estados del mundo (Brels, 2012). La propuesta de adopción de la "Declaración Universal sobre el Bienestar Animal" (OIE, 2007) constituiría un paso preliminar de importancia fundamental para abrir el camino hacia una eventual convención internacional sobre la protección del bienestar animal, estableciendo una base común sobre el principio universal de "respeto de los seres sensibles". El consenso científico es que se consideren a los perros como animales "sintientes", traducción de sentient beings, seres con sentimientos y sujetos de derechos básicos. En esta línea, son muchas las voces que refieren la necesidad de aprobar una auténtica "Declaración Universal de los Derechos de los Animales" (por ejemplo, la Fondation Droit Animal), pero desgraciadamente

\footnotetext{
${ }^{1}$ Artículos 57.12 y 281.7.

${ }^{2}$ Según el Código Sanitario para los Animales Terrestres de la Organización Mundial de Sanidad Animal (en adelante OIE) (2019), “el animal experimenta un buen bienestar si está sano, cómodo, bien alimentado, en seguridad, y si no padece sensaciones desagradables como dolor, miedo o desasosiego y es capaz de expresar comportamientos importantes para su estado de bienestar físico y mental" (artículo 7.1.1.).
}

Estudios de la Paz y el Conflicto, Revista Latinoamericana, IUDPAS-UNAH, Volumen 1, Número 1, enero-junio 2020, pp. 39-58. DOI: 10.5377/rlpc.v1i1.9515 
la aprobación de esta medida aún no ha sido efectiva y, por tanto, no cuenta con el respaldo oficial de la ONU o la UNESCO.

Lo que sí existe, y está ampliamente aceptado, especialmente por todas las sociedades veterinarias, son las cinco libertades para el bienestar animal, que son: 1) estar libre de hambre, de sed y de desnutrición; 2) estar libre de temor y de angustia; 3) estar libre de molestias físicas y térmicas; 4) estar libre de dolor, de lesión y de enfermedad; y 5) estar libre de manifestar un comportamiento natural. Asegurar que los perros, que van a hacer nuestra vida mucho mejor, tengan al menos estar cinco libertades garantizadas es una prioridad ineludible para poder llevar a cabo cualquier proyecto que los incluya con dignidad y humanidad, especialmente si dependen de las administraciones públicas, que deben marcar el ejemplo y liderar el cambio social.

En este sentido, sirve como antecedente la propuesta de "Declaración Universal de los Derechos del Animal" (aún no suscrita por la ONU), la cual estableció como principio fundamental que:

"Los animales son seres sensibles y que su bienestar debe ser respetado" (art. 1); define el "bienestar animal" como un estado positivo de bienestar (físico y psicológico) cuando el individuo está en condiciones de sanidad, libre de sufrimiento (art. 2). Asimismo, se especifica que los animales sensibles se refieren a todos los vertebrados y ciertos invertebrados teniendo la capacidad de sentimientos, incluyendo el dolor y el placer con un nivel de conciencia elevado (art. 3). Por último, se dispone la obligatoriedad de desarrollar e incorporar varios tipos de medidas adecuadas para prevenir actos crueles hacia los animales y reducir sus sufrimientos (art. 4). A fin de lograr este objetivo, deben ser desarrolladas e implementadas "políticas apropiadas, legislación y normas y todas las medidas necesarias" (LIDA, 1977).

Más recientemente, en el continente europeo, el concepto de bienestar animal está garantizado en el artículo 13 del Tratado de Funcionamiento de la Unión Europea, que reconoce a los animales como seres sensibles, con emociones, sufrimientos (dolor, temor, angustia o frustración), y también con felicidad ${ }^{3}$. Por tanto, hoy está emergiendo un nuevo paradigma que considera a los animales como seres sintientes, dejando de ser vistos como cosas, colaborando para fines y propósitos diferentes, transformado las políticas ${ }^{4}$ y fomentando la concienciación y la responsabilidad social hacia el medio en que habitamos.

Vistos todos los conceptos anteriores, ahora nos queda por ver cómo conectan todos ellos con la paz transformadora. Como afirman Montañés y Ramos (2012: 246), la paz y el conflicto son realidades socioculturales construidas, por tanto, se ha de propiciar la participación de los sujetos en la gestión de los conflictos y la construcción de la paz. El ser humano es un sistema de observación práxico (que se transforma en la acción de transformar), organizacionalmente e informacionalmente cerrado y energéticamente abierto (receptor de estímulos y emisor de energía), autopoiético (se autocrea), no trivial (capaz de innovar en las respuestas), y reflexivo (capaz de representarse a sí mismo y a otros sistemas observadores observando lo que él observa), que al acoplarse perceptivomotoramente con el

\footnotetext{
${ }^{3}$ La importancia y trascendencia del artículo 13 del TFUE, provocó un cambio en el Código Civil Francés que, no experimentaba enmiendas desde 1804, reconociendo la sintiencia animal en marzo de 2015.

${ }^{4}$ Por ejemplo, en España la acción continuada, entregada y responsable de personas que, desde hace largo tiempo, han invertido su esfuerzo, su dedicación y sus capacidades para consolidar la acción voluntaria hacia los animales se le considera "voluntariado ambiental". Persigue disminuir el impacto negativo del ser humano sobre el medio ambiente y poner en valor el patrimonio natural existente, las especies animales y vegetales, los ecosistemas y los recursos naturales realizando, entre otras, acciones de protección de los animales y cualesquiera otras que contribuyan a proteger, conservar y mejorar el medio ambiente.
}

Estudios de la Paz y el Conflicto, Revista Latinoamericana, IUDPAS-UNAH, Volumen 1, Número 1, enero-junio 2020, pp. 39-58. DOI: $10.5377 /$ rlpc.v1i1.9515 
medio y con quienes en él habitan recepciona estímulos a los que debe inferir su particular sentido, de acuerdo con la cultura hologramática insertada en su estructura organizativa. Sentidos que ha de procurar compatibilizar en las redes en las que participa para su supervivencia, pues, para que el sistema funcione y pueda sobrevivir, precisa compatibilizar las realidades construidas.

Y desde este modelo de paz transformadora, el desarrollo del Buen Vivir o Vivir Bien o Sumak Kawsay ${ }^{5}$ es fundamental para entender nuestras relaciones sociales y nuestras interacciones con el resto de seres vivos. El Buen Vivir es el proceso de la vida en plenitud, vivir en armonía con los demás seres y la naturaleza, sobre la base de la unidad, la solidaridad, la empatía y el equilibrio natural. Desde una dimensión ambiental, la construcción de paz supone respetar y cuidar la Madre Tierra porque es el soporte de la vida en el planeta. En definitiva, entender el enfoque de paz transformadora en su dimensión ambiental, y potenciarla desde la cultura del Buen Vivir, es un buen punto de partida para explorar las posibilidades de interacción mutua que existen entre los perros y los seres humanos.

Tal es así que ciertos países latinoamericanos llevan tiempo promoviendo la aprobación definitiva, en la Organización de Naciones Unidas, de una Declaración Universal sobre los Derechos de la Madre Tierra, que incluya específicamente la protección animal: "así como los seres humanos tienen derechos humanos, todos los demás seres de la Madre Tierra también tienen derechos que son específicos a su condición y apropiados para su rol y función dentro de las comunidades en los cuales existen"6. Más allá del debate filosófico y doctrinal que pueda generarse sobre el establecimiento o no del derecho de un animal, se trataría más bien de determinar los límites de la actuación humana en el aprovechamiento de esta especie (López, 2013) ${ }^{7}$. De igual modo, así como es preciso limitar el comportamiento humano hacia cualquier práctica que genere dolor, sufrimiento o daño a los animales, sería conveniente analizar y legislar aquellas prácticas que, no siendo nocivas para los animales, comporten un beneficio común para estos y los humanos, en las interacciones que puedan generarse entre ambos. Especialmente, las relaciones que se producen entre perros y humanos, a menudo resultan muy beneficiosas para las personas en ámbitos como la sanidad, la educación o la seguridad, sin causar efectos lesivos para los canes.

Como hemos visto, la paz abarca la práctica totalidad de las facetas de la vida, y del mismo modo, una gran cantidad de dichas facetas pueden verse mejoradas por la presencia o la utilización de perros. Desde hace unos años la ciencia estudia al perro desde diversas perspectivas, entre ellas la etología, o ciencia del comportamiento animal, y gracias a ello hemos ido descubriendo, con respaldo científico, multitud de ventajas que aportan los perros a la vida de las personas. En general, dada la presencia habitual de perros en nuestras calles y en nuestras vidas, es bastante sencillo y económico incorporarlos a nuestras actividades, pero esto conlleva un cambio de paradigma y garantizar el bienestar y la seguridad de dichos animales también. El perro es uno de los pocos animales que ha evolucionado para convivir con el ser humano, es uno de los pocos animales cuya convivencia con personas no sólo no es contraria a su naturaleza, sino que es deseable para el buen desarrollo de ambas especies.

\footnotetext{
${ }^{5}$ Las Constitución Políticas Bolivia (artículos 189.1, 255.I.7, 289.1, 302.I.5, 311.II.2, 349.I, 381.I) y del Ecuador incluyen este concepto en su articulado.

${ }^{6}$ Artículo 1 del Proyecto de Declaración Universal de los Derechos de la Madre Tierra.

${ }^{7}$ La autora trata esta cuestión tomando el ejemplo de los dos países aludidos, pues Alemania y Francia son los dos casos de actuación legislativa más reciente y avanzada en esta materia.

Estudios de la Paz y el Conflicto, Revista Latinoamericana, IUDPAS-UNAH, Volumen 1, Número 1, enero-junio 2020, pp. 39-58. DOI: $10.5377 /$ rlpc.v1i1.9515
} 
De modo que, ¿en qué puede favorecer el perro al humano en este proceso? De ello damos cuenta en el siguiente apartado.

\section{LA LABOR DE LOS PERROS EN LA CONSTRUCCIÓN DE PAZ: PRINCIPALES ÁMBITOS DE ACTUACIÓN}

Una vez establecido el marco teórico sobre el cual descansa nuestra investigación, es necesario puntualizar que estamos en un punto inicial en el desarrollo del bienestar animal y su aportación a la convivencia social, explicado desde la etología, esto es, desde la ciencia del comportamiento de estos seres vivos y de las relaciones que pueden llevar a cabo con los humanos. Cambiar la concepción de lo que son los animales, de lo que pueden aportan los perros a la cultura de paz transformadora desde una dimensión ambiental, es el mejor punto de inicio para entender que el mundo solo cambia si cambiamos nosotros. La curiosidad humana por comprender estas interacciones con los canes, es la precursora de la duda metodológica que antecede a la ciencia. Y ya sabemos que "la ciencia pretende encontrar la explicación verdadera de lo que pasa ahí fuera. Y la búsqueda en la ciencia, es la duda eterna" (Safina, 2016: 84).

Hemos visto que, así como es preciso limitar el comportamiento humano hacia cualquier práctica que genere dolor, sufrimiento o daño a los animales, sería conveniente analizar aquellas prácticas que, no siendo nocivas para ellos, comporten un beneficio común para los perros y los humanos. O lo que es lo mismo, ya sea como medida proactiva o reactiva, ¿en qué ámbitos de actuación pueden ser eficaces los perros para ayudar a la construcción de una sociedad mejor, más plena, feliz y en paz? Lo cierto es que son muchos los campos que pueden ser estudiados en este supuesto, pero hemos querido resumir los más relevantes en cinco grandes bloques clasificatorios: sanidad, seguridad y justicia, emergencias, salud pública y economía.

\section{Sanidad}

Recientes investigaciones dan cuenta de los efectos tan positivos que de la mera presencia de los perros genera sobre las personas en la mejora del ritmo cardiaco, la tensión arterial y los estados depresivos (Allen, Shykoff, e Izzo, 2001), con el uso de terapias asistidas por animales (Wesenberg, et al., 2012). Está ampliamente probado por varios estudios que hay resultados significativos en cuanto a mejoras del humor, de las relaciones sociales, y otros campos (Folcha, et al., 2016). También hay estudios que muestran que el mero hecho de acariciar a un perro disminuye la tensión arterial y la frecuencia cardiaca y han probado ser útiles en la disminución de los síntomas de la depresión en personas mayores que residen en centros de la tercera edad. Además, el mero hecho de poder interactuar con los perros ha demostrado tener un efecto positivo en el bienestar, la ansiedad y el humor (Ambrosi, et al., 2018) y pueden ser de gran ayuda para niños con TDAH (Schuck, et al., 2018).

Desde otra perspectiva, antes de que los síntomas negativos para la salud se manifiesten, encontramos a perros detectores de enfermedades, de modo que cada vez está más extendido el uso de perros para detectar células tumorales en pacientes en los que se sospecha de la presencia de algún tipo de cáncer (Lundqvist, et al., 2017).

Del mismo modo, se ha demostrado que hay perros capaces de detectar, con suficiente antelación, la bajada del nivel de glucosa en sangre de pacientes diabéticos, avisar antes de

Estudios de la Paz y el Conflicto, Revista Latinoamericana, IUDPAS-UNAH, Volumen 1, Número 1, enero-junio 2020, pp. 39-58. DOI: 10.5377/rlpc.v1i1.9515 
que el paciente sufra una crisis convulsiva (en el caso de personas con epilepsia) y otros canes acompañan a personas con autismo para ayudarlas a reducir ciertas crisis conductuales o de otra índole (Grajfoner, et al., 2017; Hosey, et al., 2018). Concretamente, la terapia asistida con perros en personas con Trastorno del Espectro Autista (TEA), se basa en la estimulación, a través de la interacción humano-animal para favorecer el aprendizaje individual, cumpliendo objetivos tales como el contacto visual, habilidades de comunicación verbal y no verbal, socialización en cuanto a comprensión de emociones sobre la interacción, aprendizaje y respeto, memoria, atención, habilidades de motricidad, estimulación sensorial o reducción de conductas estereotipadas y de conductas disruptivas, entre otras ${ }^{8}$.

Estos son solo algunos ejemplos de la labor tan importante que desempeñan los perros en el ámbito sanitario.

\section{Seguridad y justicia}

Este campo de actuación es uno de los más conocidos. Desde un punto de vista reactivo, es habitual que la policía y el ejército utilicen perros de defensa para proteger instalaciones críticas, así como para apoyar a los agentes y militares en sus patrullas. La capacidad de reacción del perro es superior a la de su compañero humano, por lo que en muchas ocasiones el perro habrá reaccionado ante un ataque antes de que su contraparte humana se haya dado cuenta de lo que está sucediendo, con lo que se salvarían multitud de vidas (Moreno, 2012).

En segundo lugar, los perros ayudan en la seguridad aérea. La cetrería es un servicio que requiere un equipo multidisciplinar formado por halconeros, halcones, águilas y perros. Este equipo debe patrullar todos los días el aeropuerto e implementar complejas técnicas cetreras. En algunos aeropuertos se utilizan perros para que las aves se asusten y no vuelen alrededor de las pistas de aterrizaje de los aeródromos, lo que puede causar accidentes al ser absorbidas por los motores de los aviones a propulsión, provocando que se las aeronaves se estrellen. El ejemplo más claro lo creó el médico y etólogo español, Félix Rodríguez de la Fuente, que creó este servicio que viene siendo utilizado actualmente en diversos aeropuertos españoles, como el Aeropuerto Internacional Adolfo Suárez Madrid-Barajas.

En tercer lugar, los perros detectores de drogas, explosivos, municiones, dinero, etc. son, probablemente, una de las especialidades más conocidas. Tal es así que raro es el cuerpo policial o militar en el mundo que no utilice perros en su servicio diario (Mackinnon, 2017).

En cuarto lugar, desde la criminalística, empiezan cada vez a ser más utilizados los perros detectores de huellas odoríficas en la escena del crimen. Países como Cuba u Holanda usan perros especialmente adiestrados para detectar "huellas odoríficas". En esta técnica, la huella no es más que una muestra de olor que el perro identifica y relaciona con alguien que estuvo en la escena del crimen cometido. Incluso diferentes tribunales de justicia ya lo aceptan como prueba irrefutable (Hernández, 2003).

En el campo de la justicia, llevar perros de acompañamiento en declaraciones judiciales sigue siendo algo relativamente novedoso en el resto del mundo ${ }^{9}$. Sin embargo, en Chile y

\footnotetext{
${ }^{8}$ Como ejemplo ilustrativo, Bocalán Argentina es una Organización sin fines de lucro que trabaja en Interacciones Asistidas con Animales (IAA) y entrenamiento y entrega de perros de asistencia.

${ }^{9}$ En España, desde septiembre de 2014, los Juzgados 23, 25, 27, 29 y a la Audiencia Provincial de Familia de la Comunidad de Madrid, cuentan en su plantilla con la figura del Perro de Apoyo a Menores en Juzgados. Así mismo, en julio de 2016, el Principado de Asturias, empezó a implantar un programa dirigido a menores incursos en procesos judiciales, como víctimas o testigos, en los órdenes penal o civil, incluyendo también la

Estudios de la Paz y el Conflicto, Revista Latinoamericana, IUDPAS-UNAH, Volumen 1, Número 1, enero-junio 2020, pp. 39-58. DOI: $10.5377 /$ rlpc.v1i1.9515
} 
Argentina es más habitual que las personas que deben acudir a declarar en los juzgados puedan ir acompañadas de perros de asistencia para permanecer más tranquilos mientras pasan por una situación que, en general, resulta bastante estresante. Colectivos específicos tan castigados como la infancia o las víctimas de violencia de género, encuentran en los peludos los mejores aliados para ganar confianza y seguridad en sí mismas.

\section{Emergencias}

Los perros de búsqueda y rescate son fundamentales en situación de riesgo colectivo sobrevenidas por un evento que pone en peligro inminente a personas o bienes y exige una gestión rápida por parte de los poderes públicos para atenderlas y mitigar los daños y tratar de evitar que se convierta en una catástrofe ${ }^{10}$. Esta definición de emergencia se corresponde con otras denominaciones como emergencia extraordinaria, por contraposición a emergencia ordinaria que no tiene afectación colectiva. Terremotos, personas perdidas, aludes, cadáveres en el agua, etc. Por desgracia, continuamente vemos cómo los perros ocupan portadas de diarios internacionales al ser quienes encuentra supervivientes que llevan varios días sepultados por los escombros después de un corrimiento de tierras o un terremoto, en la búsqueda de personas perdidas en el campo o la montaña, o en la búsqueda de cadáveres sumergidos en el agua, tarea en la que también destacan ayudando a encontrar cuerpos de personas que se han ahogado en embalses o $\operatorname{lagos}^{11}$.

\section{Salud pública}

Otro de los ámbitos de actuación de los perros es en la salud pública. Los perros detectores de plagas, chinches, hongos puedes ser fundamentales para mitigar los problemas de inseguridad ambiental o alimentaria que se producirán masivamente en años próximos. En muchos lugares hay infestaciones de diversos tipos que afectan a hogares, lugares de trabajo, escuelas, hospitales o a la propia naturaleza. El olfato del perro es una gran ayuda para detectar precozmente los lugares que empiezan a estar afectados de estos parásitos para poder actuar sobre el problema antes de que sea irresoluble o provoque grandes pérdidas sociales y económicas, provocando incluso desplazamiento de familias enteras, cuando no de localidades completas ${ }^{12}$, para evitar u contagio.

presencia de canes en separaciones o divorcios, entrega en puntos de encuentro, procesos de adopción, etc., así como a otras víctimas de delitos violentos y violencia de género.

\footnotetext{
${ }^{10}$ Así se define emergencia en el artículo 2.5 de la Ley 17/2015, de 9 de julio, del Sistema Nacional de Protección Civil en España.

${ }^{11}$ Un ejemplo es la Escuela Española de Salvamento y Detección con Perros (ESDP), como organización internacional, que fue fundada en 1995 por un grupo de profesionales, dedicados al trabajo con perros de búsqueda y salvamento en sus diferentes especialidades. Han participado en multitud de intervenciones dentro y fuera del territorio nacional, formando parte de los contingentes de ayuda de emergencia que el estado español ha enviado a los terremotos de Turquía (agosto y noviembre 1999), El Salvador (enero y febrero 2001), Argelia (2003), Marruecos (2004) y Haití (2010), Ecuador (2016).

${ }^{12} \mathrm{La}$ Universidad de Melbourne (Australia) entrena a perros detectores de plagas y enfermedades en los viñedos, trabajando en la filoxera, un insecto que se alimenta de la raíz de viñedos. En Estados Unidos, los perros han ayudado a detectar la chinche hedionda o stink bug, que causaba graves problemas en hogares y
}

Estudios de la Paz y el Conflicto, Revista Latinoamericana, IUDPAS-UNAH, Volumen 1, Número 1, enero-junio 2020, pp. 39-58. DOI: $10.5377 /$ rlpc.v1i1.9515 
Otro ejemplo lo constituyen los perros guía. Sin duda, la cara más visible de los perros de asistencia es el perro de acompañamiento para personas ciegas -también denominado perro lazarillo-. Mucha gente no podría hacer una vida normal sin la ayuda de estos animales. Los perros de asistencia en determinadas enfermedades conforman otra gran categoría de actuación. Del mismo modo que el perro guía cumple su función, se está popularizando la labor desempeñada por el perro de asistencia, que incluye desde el acompañamiento a alguien incapaz de valerse por sí mismo en todas las facetas de la vida y es auxiliado por el perro, hasta el acompañamiento y apoyo emocional -aunque estos últimos son más raros de ver en el mundo latinoamericano-.

En este sentido y por aportar mayor comprensión del supuesto planteado, recientemente se ha aprobado una normativa española ${ }^{13}$ que clasifica a los perros de asistencia en cinco grupos, todos ellos esenciales en las relaciones de los canes con los seres humanos: a) perro guía, adiestrado para guiar a una persona con discapacidad visual, ya sea total o parcial, o con una discapacidad auditiva añadida; b) perro de servicio, adiestrado para promover la autonomía personal de una persona con discapacidad física, mediante la ayuda y asistencia en las actividades de la vida diaria; c) perro de señalización de sonidos, adiestrado para avisar a las personas con discapacidad auditiva de la emisión de sonidos y su procedencia; d) perro de aviso, adiestrado para dar una alerta médica a las personas que padecen diabetes, epilepsia u otra enfermedad; y e) perro para personas con trastorno del espectro autista, adiestrado para promover la autonomía personal de estas personas usuarias mediante la ayuda y asistencia en las actividades de la vida diaria.

Por último y como ámbito más novedoso de actuación dentro de este campo, podemos encontrar a perros para facilitar la convivencia y la reinserción de reclusos en la sociedad, mejorar la conducta de las personas, su autoestima, el estado de ánimo, el desarrollo de sus habilidades sociales y el control de la agresividad ${ }^{14}$. Sin duda, un avance para lograr la efectiva incorporación de estas personas a la sociedad.

\section{Economía}

Desde otra perspectiva, los perros de caza son una de las razas usadas para la actividad cinológica, que abarca desde perros cazadores que capturan a las presas, hasta los recuperadores que se encargan de ir a buscarlas.

En segundo lugar, los perros detectores de setas o trufas suponen una fuente de ingresos muy importante para mucha gente (particulares, autónomos o empresas), dedicadas profesionalmente a su recolección y que implican un alto valor porque las trufas crecen bajo tierra y pueden ser percibidas por los canes. Los perros también son grandes detectores de Boletus Edulis.

campos de cultivo. A otro nivel, en la industria maderera de almacenamiento, es imposible ver desde el exterior si el paquete de madera está en buenas condiciones, siendo los perros los mejores detectores de hongos.

${ }^{13}$ Según se establece en el artículo 5 de la Ley 11/2019, de 3 de abril, de acceso al entorno de las personas usuarias de perro de asistencia en la Comunidad de Castilla y León.

${ }^{14}$ La cárcel de Valdemoro de Madrid (España), ofrece un programa con animales como medida para reducir la ansiedad y mejorar la relación entre los reclusos. A su vez, la Fundación Affinity empezó a trabajar en prisiones en 1993, introduciendo dos perros en el centro penitenciario de Brians (Barcelona). En 2008, esta Fundación firmó un convenio con Instituciones Penitenciarias para implementar un programa de terapias más amplio y en más centros, y desde esa fecha hasta hoy, más de 4.500 reclusos de 14 prisiones españolas han podido convivir con más de 50 perros del programa y mejorar a nivel psicosocial.

Estudios de la Paz y el Conflicto, Revista Latinoamericana, IUDPAS-UNAH, Volumen 1, Número 1, enero-junio 2020, pp. 39-58. DOI: 10.5377/rlpc.v1i1.9515 
También encontramos perros detectores de acelerantes, para empresas aseguradoras de bienes y equipos; perros detectores de escapes de líquidos o gases, para empresas donde el control de este tipo de fugas sea primordial para su seguridad o seguridad ambiental; perros detectores de altas o bajas concentraciones de determinado químico, para empresas certificadoras de calidad o empresas que subcontraten la fabricación de productos acordes a unos patrones determinados de composición; erros detectores de veneno, para cuerpos policiales encargados de la naturaleza que buscan cebos envenenados; o perros pastores/mastines protectores de ganado, que evitan el robo del ganado y su pérdida causada por el ataque de animales salvajes.

Los ejemplos planteados son muy diversos y estos son solo algunos de los campos de actuación en los que la labor de los perros resulta fundamental para asegurar modelos de vida convivencial en paz, transformando nuestra realidad. Si planteamos un nuevo enfoque en su concepción y práctica, los perros garantizan al ser humano un aprendizaje, una socialización, una convivencia, una predisposición para que los comportamientos que adoptemos con ellos sean los más exitosos posibles, para que alcancemos el máximo nivel de bienestar en la interacción que establezcamos con estos seres vivos.

El conflicto (ya sea de tipo social, cultural, económico o de cualquier otra índole), sólo existe entre seres humanos y es producto de las acciones humanas. "Si un perro muerde a un ser humano, es probable que el mordisco le genere malestar, pero nunca generaría un conflicto con el perro, si acaso sólo con su amo. Un sistema no humano jamás constituye un conflicto, de modo que un perro jamás podría llegar a considerar que lo que él ve, lo están viendo otros seres porque no es reflexivo" (Ramos, 2015: 106). Pero sí es un ser sintiente. Por eso, bajo el modelo de paz transformadora, debemos asumir que sólo a nosotros (los humanos), nos corresponde generar esas sinergias mutuas, esas reflexiones con otros seres sintientes (los perros), que refuercen modos de convivencia que sumen a ambos y que no resten; donde se entienda que el estudio del comportamiento de los animales -en nuestro caso, de los perros-, genera amplios beneficios para quienes se relacionan con ellos (personas); y que entender su bienestar y el nuestro pasa por rechazar y castigar lo que causa sufrimiento o dolor a estos seres vivos, pero también por asumir y garantizar el papel tan relevante que pueden desempeñar en facetas esenciales de nuestra vida.

\section{ALGUNOS AVANCES LATINOAMERICANOS EN LA MATERIA}

Una vez vista la parte más teórica sobre la aportación de los perros al mantenimiento y consecución de la paz en Latinoamérica, conviene aterrizar sobre el terreno y observar qué están haciendo los estados o cómo están regulando las prácticas que permitan el reconocimiento de ciertos derechos a estos animales para gratificar sus servicios con la dignidad que merecen.

Desde hace algunos años, emerge un nuevo patrón cultural de rechazo a los malos tratos hacia los animales, activándose más denuncias sobre actos crueles e inhumanos hacia estos seres vivos. A su vez, la tendencia a adoptar perros es cada vez mayor ${ }^{15}$, incrementando con

\footnotetext{
${ }^{15}$ No hemos encontrado evidencia empírica para el caso Latinoamericano, pero en España, tener un animal en el entorno urbano es cada vez más frecuente. Según datos de 2015 del periódico Expansión, existen en España, 20 millones de mascotas, de los cuales 7,3 millones corresponden a perros y gatos (5.147.000 perros y 2.265.000 gatos). Esto quiere decir que, aproximadamente, 4 de cada 10 hogares posee al menos una mascota. Es

Estudios de la Paz y el Conflicto, Revista Latinoamericana, IUDPAS-UNAH, Volumen 1, Número 1, enero-junio 2020, pp. 39-58. DOI: 10.5377/rlpc.v1i1.9515
} 
ello los modelos convivenciales entre seres humanos y seres no humanos. Pero, ¿a qué se debe este cambio de percepción social? Son muchos los factores que explican este hecho, entre ellos, los beneficios psicológicos, fisiológicos y sociales que aporta la tenencia de los animales de compañía. Vamos a exponer algunos ejemplos que explicar cuál es la realidad latinoamericana en este aspecto.

Partiendo del reconocimiento normativo, hay países latinoamericanos extremadamente avanzados al respecto, como es el caso de México, que incluye la protección de los animales en su Carta Magna: "esta Constitución reconoce a los animales como seres sintientes y, por lo tanto, deben recibir trato digno. (...). Su tutela es responsabilidad común (...)”. Otros muchos estados también reconocen y regulan la protección animal, aunque no se garantiza este derecho con la efectividad que merece.

Si analizamos el caso concreto de países centroamericanos ${ }^{16}$, Costa Rica comenzó a legislar a favor del bienestar y la protección de los animales en el año 1996. Posteriormente, lo hizo Nicaragua en 2010 y, a partir de ese momento, fueron muchos los estados que se han ido sumando al reconocimiento de los animales domésticos y silvestres domesticados, como seres capaces de sentir, percibir y reaccionar frente a la felicidad o el sufrimiento. En concreto, los ordenamientos jurídicos de Nicaragua, Honduras, El Salvador y Guatemala garantizan el bienestar a otros seres vivos no humanos. Por tanto, el bienestar individual de cada animal cuenta y encaminar a la sociedad a actuar en beneficio de todo ser vivo -también de los perros- es una labor fundamental que hay que promover y respetar.

En base a lo anterior, podemos afirmar que existe una moderna tendencia jurídica a regular, de forma expresa y tácita, la protección y el bienestar animal en muchas leyes de los estados latinoamericanos, incluyendo en todas ellas las cinco libertades básicas del bienestar animal citadas con anterioridad ${ }^{17}$, con similitudes legislativas que pueden apreciarse claramente. Sin embargo, esta regulación no ha surtido los efectos esperados, bien por la falta de voluntad de los gobiernos (Arguello, 2017), bien por la propia incomprensión del término "sintiencia animal" que condiciona su propia aplicación, bien por la constante crisis de institucionalidad en el incumplimiento sistemático de la ley que ocurre con frecuencia en estados hermanos más allá del charco. Al reconocimiento formal debe seguirle su aplicación efectiva $^{18}$, del tal modo que esto se convierta en un verdadero mecanismo para el cambio social.

interesante analizar también el principal motivo por el que una familia decide tener un animal: a) por el gusto por los animales $(48,4 \%)$; b) porque le hace compañía $18,7 \%)$; y c) por hacer un regalo $(9,6 \%)$. De este modo, podríamos decir que la tenencia de animales de compañía responde a sentimientos $(49,1 \%$ de los casos), a beneficios $(21,4 \%$ de los casos) y a obsequios (16,1\% de los casos). Link con acceso a la noticia: https://www.expansion.com/directivos/estilo-vida/2018/03/16/5aab908cca4741a1098b4575.html.

${ }^{16}$ Conforme a: Decreto 05-2017 que aprueba la Ley de Bienestar Animal Guatemala; Decreto 115- 2015 que aprueba la Ley de Protección y Bienestar Animal de Honduras; Ley 330 de Protección y Promoción del Bienestar de Animales de El Salvador; Ley para la Protección y Bienestar de los Animales Domésticos y Silvestres Domesticados de Nicaragua; Ley de Bienestar de los Animales de Costa Rica; y Ley 70 de 12 de octubre de 2012, de protección de animales domésticos de Panamá.

${ }^{17}$ Libre de hambre, sed y desnutrición; libre de miedos y angustias; libre de incomodidades físicas o térmicas; libre de dolor, lesiones o enfermedades; y libre para expresar las pautas propias de comportamiento.

${ }^{18}$ Sirva como ejemplo el hecho de que, en numerosos textos constitucionales latinoamericanos, la dignidad humana se regula como un derecho fundamental de la ciudadanía. Sin embargo, la laxitud del concepto, así como la complejidad normativa, hacen poco efectivo que la persona humana sea el fin supremo de la sociedad y del Estado, a la que todos tienen la obligación de respetarla y protegerla, siendo esta inviolable (artículo 59 de la Constitución de Honduras, a título ilustrativo).

Estudios de la Paz y el Conflicto, Revista Latinoamericana, IUDPAS-UNAH, Volumen 1, Número 1, enero-junio 2020, pp. 39-58. DOI: 10.5377/rlpc.v1i1.9515 
Si a todo lo expuesto añadimos el hecho de que estos nuevos conceptos son aún vistos con prudencia y desconocimiento por la sociedad, de que las instituciones no realizan esfuerzos para consagrarlos en la sociedad, de que no se ha incorporado un exhaustivo estudio del actual ordenamiento normativo de los estados antes de iniciar los motores de oportunidad legislativa o de que siguen sin crearse tutores de la política pública sobre el bienestar animal, aún son muchos los pasos que deben darse para naturalizar nuestras interacciones con los animales para reportarnos un beneficio mutuo.

Eso desde el punto de vista jurídico y sólo exponiendo algunos ejemplos. Si observamos los mecanismos públicos que se están activando para generar una culta de paz transformadora desde la dimensión ambiental y relacionándonos con los canes, en Bolivia está vigente desde 2009 una ley que prohíbe el uso de animales silvestres y domésticos en circos, por considerar que en esos espectáculos se practican "actos de crueldad" hacia ellos. Seis años más tarde, el gobierno boliviano aprobó otra ley para prevenir y sancionar el maltrato de animales domésticos, aunque sigue permitiendo su uso y sacrificio en medicina tradicional y rituales ancestrales.

En Argentina ha acontecido un hecho singular, pues han llegado a reconocer a una orangutana como sujeto titular de derechos ${ }^{19}$, lográndose su libertad y ordenando la justicia su traslado a un centro adecuado para sus necesidades específicas. Así, en marzo de 2015, el asunto llegó al Juzgado Contencioso, Administrativo y Tributario número 4 de la Ciudad de Buenos Aires que, tras una denuncia contra el cautiverio de este animal, la juez consideró a la orangutana Sandra sujeto de derecho (no objeto) y ordenó al propietario del zoológico garantizar al animal "las condiciones naturales del hábitat y las actividades necesarias para preservar sus habilidades cognitivas". Esto entronca con la famosa Declaración de Cambridge sobre la Conciencia, en la que se proclamó que: "los animales no humanos poseen substratos neuroanatómicos, neuroquímicos y neurofisiológicos de los estados de consciencia, junto con la capacidad de exhibir comportamientos intencionales" (Low, 2012). Esta Declaración ha marcado un antes y un después en la sintiencia animal.

Por otra parte, y tal y como ya hemos tenido ocasión de exponer, en Chile y también en Argentina -además de Canadá, EEUU, Irlanda o España-, los perros acompañan a las víctimas en el juzgado para que puedan declarar ante los jueces de una manera menos estresante $^{20}$. Si atendemos solamente a las declaraciones de abusos sexuales en menores, los datos disponibles en Europa sugieren que aproximadamente 1 de cada 5 niños son víctimas de alguna forma de violencia sexual. Entre ellos, se estima que en el $70 \%$ a $85 \%$ de los casos el abusador es alguien que el niño conoce y en quien confía ${ }^{21}$. Introducir el apoyo de los perros en los juzgados para ayudar en las declaraciones de las víctimas, puede hacer posible el objetivo no ya sólo de hacer justicia, sino también de humanizar todo el proceso judicial.

Otro ejemplo. En Colombia, la policía cuenta con patrullas de protección animal, cuya función especial consiste en apoyar a las autoridades, en los casos de maltrato animal y con la incautación de vida silvestre objeto de tráfico ilegal, pasando a disposición de autoridades

\footnotetext{
${ }^{19}$ Esta noticia fue ampliamente difundida por los medios de comunicación, entre ellos El País. Link de acceso: https://elpais.com/elpais/2019/06/17/eps/1560778649_547496.html.

${ }^{20}$ La iniciativa de llevar a perros de asistencia a los juzgados nació en Seattle (Estados Unidos), en el año 2003, con la fiscal Ellen O'Neill-Stephens para casos relacionados con drogas. Tras el éxito de la propuesta, el programa se amplió a víctimas de abuso sexual y violencia intrafamiliar en menores. Po ejemplo, en Chile se ha probado que en un $90 \%$ de los casos en los que se emplean perros, se consiguen relatos y testimonios Links con acceso a estas noticias: https://bit.ly/2klobxf; https://bit.ly/2lEsuns; https://bit.ly/2m5JoM4.

${ }^{21}$ Datos ofrecidos por el Consejo de Europa, en "Campaign to stop sexual violence against children" (2014).
}

Estudios de la Paz y el Conflicto, Revista Latinoamericana, IUDPAS-UNAH, Volumen 1, Número 1, enero-junio 2020, pp. 39-58. DOI: 10.5377/rlpc.v1i1.9515 
ambientales $^{22}$. Incluso la Alcaldía de Bogotá ha creado un Instituto Distrital de Protección y Bienestar Animal, como entidad rectora de la protección y bienestar de la fauna doméstica y silvestre a través de la atención integral, la promoción de una cultura ciudadana basada en un solo bienestar humano-animal y la participación ciudadana en la construcción de una sociedad corresponsable y sensible con la vida y el trato digno a los animales ${ }^{23}$. Además, a esta medida se unen las elevadas multas con sanciones tipificadas por actos de crueldad y violencia contra los animales, ya que la cifra puede ascender a 60 salarios mínimos mensuales del país ${ }^{24}$, con penas de prisión que oscilan entre los 12 y los 36 meses.

En Ecuador también se sumaron con la creación en 2016 de la oficina de Estudios de la Relación entre Animales y Humanos (ERAH), perteneciente a la Dirección de Bienestar Animal y Gestión Social de la Prefectura del Guayas. El ERAH es el primer organismo del mundo que ha desarrollado políticas públicas de protección animal, desde un punto de vista antrozoológico. A su vez, en una consulta popular en 2011, Ecuador decidió prohibir espectáculos públicos donde se produzca la muerte de un animal. 110 municipios se acogieron, en este mismo año, a iniciativa impulsada por el Gobierno y prohibieron las corridas de toros, aunque pueden organizarse festejos sin la muerte del animal.

En Uruguay se ha creado la Comisión Nacional Honoraria de Tenencia Responsable y Bienestar Animal, a fin de realizar o fomentar investigaciones y estudios relacionados con la situación de los animales, su comportamiento y su protección ${ }^{25}$. La ley contra el maltrato animal establece sanciones de hasta dos años de cárcel para quien mate a un animal doméstico y multas de más de dos mil dólares por maltrato. Además, en ciertos casos, también prohíbe los circos con animales y los zoológicos.

Desde abril de 2015, Perú elevó las penas por maltrato animal con condenas de hasta 5 años de cárcel, y la máxima se aplica por matar animales domésticos y/o silvestres que se encuentren en cautiverio. Su legislación también estipula una pena máxima de dos años por maltratar o someter a los animales a realizar actividades ajenas a su propia naturaleza.

Los casos expuestos tan sólo dan cuenta de cómo Latinoamérica está afrontando esta cultura de paz transformadora en su dimensión ambiental, dando un nuevo paso hacia el reconocimiento del bienestar animal en sus textos normativos y en sus políticas públicas. El aprovechamiento consciente de este vínculo humano-animal, mediante la aplicación terapéutica del mismo para por otorgar a estos seres vivos no humanos en lugar que merecen. Crear nuevos modelos y formas convivenciales (paz transformadora), bajo el paradigma básico del Buen Vivir, entendiendo que los perros son seres sintientes (sintiencia animal), y que estudiar su comportamiento (etología) puede reportar avances significativos al progreso y bienestar general en marco de construcción colectiva de sinergias y oportunidades, es el primer paso para afrontar con éxito medidas institucionales y prácticas que, no siendo nocivas para los animales, comporten un beneficio común para estos y los humanos, en las interacciones que puedan generarse entre ambos. En el caso de los perros, el objetivo debe ser su reconocimiento, su protección, la creación de refugios, la inclusión de los delitos contra los animales en los códigos penales y la organización y estandarización del trabajo de los perros en cualquiera de las facetas de la vida en la que actúan.

\footnotetext{
${ }^{22}$ Link de acceso a este servicio: https://www.policia.gov.co/proteccion-ambiental/patrullas.

${ }^{23}$ Link de acceso a este servicio: http://www.proteccionanimalbogota.gov.co/.

${ }^{24}$ En 2019, el salario mínimo mensual era de aproximadamente 247 dólares.

${ }^{25}$ Artículo 16 D de la Ley No 18.471 , de Tenencia Responsable de Animales, de la República Oriental del Uruguay.
}

Estudios de la Paz y el Conflicto, Revista Latinoamericana, IUDPAS-UNAH, Volumen 1, Número 1, enero-junio 2020, pp. 39-58. DOI: $10.5377 /$ rlpc.v1i1.9515 
Tal vez algún día avancemos hasta considerar a los animales como auténticos seres sintientes y aprendamos, con su comportamiento, a modular el nuestro. Desde una dimensión ambiental de paz transformadora, los perros son los mejores aliados para lograr más progreso y bienestar. Porque,

"Comprender al resto de animales no es ningún afán de moda. Si no lo logramos, aceleraremos la quiebra y el fin de nuestro planeta. Y si tratásemos a los animales como se merecen, la inhumanidad humana hacia los humanos despuntaría de forma aún más terrible. Podríamos dirigir nuestra atención al paso siguiente más allá de la civilización humana: la civilización humanitaria. Justicia para todos" (Safina, 2016: 472).

Construir paz es también reconocer a otros seres vivos y transformar nuestra existencia con su ejemplo.

\section{CONCLUSIONES}

La paz sólo se comprende como un constructo social en permanente transformación que se desarrolla cuando logramos cristalizar modelos de vida en nuestras, comunidades, sociedad y sistemas políticos y económicos que facilitan el desarrollo de las potencialidades humanas del conjunto de la población. Es por ello que se afirma que la paz es el camino (y no la meta) que construimos los seres humanos participativamente cuando nos comprometemos al desarrollo del Buen Vivir. Y desde este modelo de paz transformadora, es fundamental para entender nuestras relaciones sociales y nuestras interacciones con el resto de seres vivos. Desde una dimensión ambiental, la construcción de paz supone respetar y cuidar la Madre Tierra porque es el soporte de la vida en el planeta. Entender el enfoque de paz transformadora en su dimensión ambiental, y potenciarla desde la cultura del Buen Vivir, es un buen punto de partida para explorar las posibilidades de interacción mutua que existen entre los animales y los seres humanos, entre los perros y las personas.

La tendencia social generalizada avanza hacia el reconocimiento de los derechos a la naturaleza, a los animales, desde preceptos de igualdad universal, dejando a un lado esa creencia de que el ser humano es el único ser capaz de sentir y expresar sentimientos, emociones, felicidad y dolor. Pero encontramos diferentes escenarios de aplicación normativa y limitaciones que impiden esclarecer ante qué situaciones se pueda aplicar adecuadamente dichos marcos reguladores. Es urgente que la Comunidad Internacional debe reconocer como tal la Declaración Universal de los Derechos de los Animales, así como formular campañas públicas de concienciación hacia el bienestar animal y su reconocimiento como seres sintientes, $y$, por lo tanto, sujetos a derecho.

Es notoria la importancia de los animales en todas las sociedades humanas pero, concretamente, las Latinoamericanas han demostrado su capacidad de ir más allá y legislar para garantizar su bienestar, incluso a nivel constitucional, en leyes federales y en sus códigos penales. Sin embargo, el camino no termina con el reconocimiento formal del derecho, sino con la práctica diaria, con la implementación de las políticas públicas y con la concienciación social sobre la importancia de incluir la sintiencia animal en nuestra realidad actual. Latinoamérica ha dado el pistoletazo de salida y tiene ahora una oportunidad única de liderar un cambio a nivel mundial, postulándose como garante de los derechos de los seres que tanto hacen por construir paz en las sociedades de todo el mundo. Para ello se debe regular apropiadamente la relación entre los seres humanos y los animales, entre las personas y los

Estudios de la Paz y el Conflicto, Revista Latinoamericana, IUDPAS-UNAH, Volumen 1, Número 1, enero-junio 2020, pp. 39-58. DOI: 10.5377/rlpc.v1i1.9515 
perros, especialmente en los casos en los que usamos los servicios de los canes para el bien común dado que, en muchas de esas actividades tan provechosas para nosotros, los animales afrontan algún riesgo que es necesario prever y evitar.

Los perros son verdaderos impulsores y constructores de paz. Estudiar y comprender su comportamiento puede ayudarnos a generar transformaciones sociales que se manifiesten positivamente en muchos ámbitos de la vida y en políticas esenciales de cualquier estado. Se hace necesario, además de limitar la actuación humana hacia cualquier práctica que genere dolor, sufrimiento o daño a los canes, legislar aquellas prácticas que, no siendo nocivas para ellos, comporten un beneficio común para estos y los humanos, en las interacciones que puedan generarse entre ambos.

\section{REFERENCIAS}

Allen, K., Shykoff, BE., e Izzo JL. (2001). "Pet ownership, but not ace inhibitor therapy, blunts home blood pressure responses to mental stress", Hypertension, 38:4, 815-20.

Ambrosi, C., Zaiontz, C., Peragine, G., Sarchi, S., Bona, F. (2018). "Randomized controlled study on the effectiveness of animal-assisted therapy on depression, anxiety, and illness perception in institutionalized elderly", Psychogeriatrics, 19(1), 55-64. (Disponible online: https://onlinelibrary.wiley.com/doi/full/10.1111/psyg.12367).

Arango, V. (2007). Paz social y Cultura de Paz. Panamá: Ediciones Panamá Viejo. (Disponible online: http://www.corteidh.or.cr/tablas/30445.pdf)

Arguello, A. (2017). "Situación jurídica de los animales en Centro América como seres sintientes ("sentientes beings")", Derecho Animal: Forum of Animal Law Studies, 8-3, 112. (Disponible online: https://doi.org/10.5565/rev/da.7).

Brels, S (2012). "La protección del bienestar animal: una preocupación universal que se debe considerer globalmente y seriamente en Derecho Internacional", Derecho Animal, 3: 2. (Disponible online: https://doi.org/10.5565/rev/da.178)

Broom D.M. (2006). Animal Welfare. Strasbourg: Council of Europe Editions, 137-169.

Conferencia de los Pueblos sobre el Cambio Climático y los Derechos de la Madre Tierra (2010). Proyecto de Declaración Universal de los Derechos de la Madre Tierra. Cochabamba, Bolivia: Ministerio de Culturas.

Dawkins, M.S. (1993). Through Our Eyes Only? The Search for Animal Consciousness. Oxford: W.H. Freeman.

Decreto 05-2017 que aprueba la Ley de Bienestar Animal Guatemala.

Decreto 115- 2015 que aprueba la Ley de Protección y Bienestar Animal de Honduras. Dossier Legislativo de Protección de Animales de la Biblioteca del Congreso de Argentina. Año III, $n^{\circ} 86$, septiembre de 2015.

Mackinnon, P. (2017). Detector dog: A Talking Dogs Scentwork Manual. London: Hubble \& Hattie.

Montañés, M. y Ramos, EA. (2012). "La paz transformadora: una propuesta para la construcción participada de paz y la gestión de conflictos desde la perspectiva sociopráxica", OBETS, Revista de Ciencias Sociales, 7: 2, 241-269. (Disponible online: http://dx.doi.org/10.14198/OBETS2012.7.2.04).

Moreno, F.J. (2012). "Perros especialistas en guarda, defensa y acompañamiento". En Francisco Salvador Miguel (Coord.), Investigación de la educación y adiestramiento canino orientado a tareas de seguridad y asistencia. Madrid: Dykinson, 331-373.

Estudios de la Paz y el Conflicto, Revista Latinoamericana, IUDPAS-UNAH, Volumen 1, Número 1, enero-junio 2020, pp. 39-58. DOI: $10.5377 /$ rlpc.v1i1.9515 
Folcha, A., Torrente, M., Heredia, L., Vicens, P. (2016). "Effectiveness of dog-assisted therapy in the elderly. A preliminary study", Revista Española de Geriatría y Gerontología, 51: 4, 210-216. (Disponible online: https://doi.org/10.1016/j.regg.2015.12.001).

Grajfoner, D., Harte, E., Potter, LM., McGuigan, N. (2017). "The Effect of Dog-Assisted Intervention on Student Well-Being, Mood, and Anxiety", International Journal of Environmental Research and Public Health, 14: 5. (Disponible online: https://doi.org/10.3390/ijerph14050483).

Hernández, MB y Fuentes, VM. (2018). "La Ley Orgánica de Bienestar Animal (LOBA) en Ecuador: análisis jurídico", Derecho Animal (Forum of Animal Law Studies), 9/3, pp. 108126.

Hernández, R. (2003). "La odorología criminalística en Cuba”, Revista Cubana de Derecho, 41, 87-104.

Hosey, MM., Jaskulski, J., Wegener, S., Chlan, L., Needham, D. (2018). “Animal-assisted intervention in the ICU: a tool for humanization", Critical Care, 22: 22. (Disponible online: https://doi.org/10.1186/s13054-018-1946-8)

LIDA (Liga Internacional de los Derechos del Animal) (1977). Declaración Universal de los Derechos del Animal, adoptado en la $3^{\circ}$ Reunión sobre los Derechos del Animal. Londres: Liga Internacional de los Derechos del Animal.

López, P. (2013). "La protección jurídica de los animales en Francia y Alemania", Revista Aranzadi de Derecho Ambiental, $\mathrm{n}^{\circ} 25,281-291$.

Low, P. (2012). Declaración de Cambridge sobre la Conciencia en Animales Humanos y no Humanos. Cambrigde: Universidad de Cambridge.

Lundqvist, M., Carlsson, P., Sjödahl, R., Theodorsson, E., Levin, L. (2017) "Patient benefit of dog-assisted interventions in health care: a systematic review", BMC, Complement Alternative Medicine, 17(1), 358. (Disponible online: https://doi.org/10.1186/s12906017-1844-7)

Ramos, E. (2015). “La paz transformadora: una paz integral y participativa”, Revista Pueblos, $n^{\circ}$ 57, Dossier Construcción de Paz.

Ramos, E. (2016). Paz transformadora (y participativa). Teoría y método de la paz y el conflicto desde la perspectiva sociopráxica. Tegucigalpa: Instituto Universitario en Democracia, Paz y Seguridad de la Universidad Nacional Autónoma de Honduras (IUDPASUNAH). (Disponible online: https://iudpas.unah.edu.hn/dmsdocument/2738-paztransformadora-y-participativa).

Safina, C. (2016). Beyond words. What Animals Think and Feel. Barcelona: Galaxia Gutenberg.

Schuck, SEB., Johnson, HL., Abdullah, MM., Stehli, A., Fine AH., Lakes KD. (2018). “The Role of Animal Assisted Intervention on Improving Self-Esteem in Children With Attention Deficit/Hyperactivity Disorder", Front Pediatric, 2: 6, 300. (Disponible online: https://doi.org/10.3389/fped.2018.00300)

OIE (Organización Mundial de Sanidad Animal) (2007). Declaración Universal sobre el Bienestar Animal. Paris: Comité Internacional de la Organización Mundial de Sanidad Animal (Disponible en https://www.oie.int/doc/ged/D4080.PDF).

OIE (2019). Código Sanitario para los Animales Terrestres (28 a edición). Paris: Organización Mundial de Sanidad Animal.

Estudios de la Paz y el Conflicto, Revista Latinoamericana, IUDPAS-UNAH, Volumen 1, Número 1, enero-junio 2020, pp. 39-58. DOI: 10.5377/rlpc.v1i1.9515 
ONU (Organización de Naciones Unidas) (1999). Declaración y Programa de Acción sobre una Cultura de Paz. A/RES/53/243 6 de octubre de 1999. (Disponible online: http://decade-culture-of-peace.org/resolutions/resA-53-243-esp.pdf)

ONU (2005). Informe A/59/2005 del Secretario General, Un concepto más amplio de la libertad: desarrollo, seguridad y derechos humanos para todos. Disponible en https://www.un.org/spanish/largerfreedom/report-largerfreedom.pdf

ONU (2012). Declaración A/66/750, de 20 de marzo de 2012, sobre Sociedades sostenibles, ciudadanos responsables, aprobada en la $64^{\mathrm{a}}$ Conferencia Anual del Departamento de Información Pública para las Organizaciones No Gubernamentales, celebrada en Bonn (Alemania), el 5 de septiembre de 2011. (Disponible en https://undocs.org/pdf?symbol=es/A/66/750).

PNUD (Programa de las Naciones Unidas para el Desarrollo) (1994). Human Development Report 1994. Nueva York: Programa de las Naciones Unidas para el Desarrollo.

Tribunal de Cuentas Europeo (2018). "Bienestar animal en la UE: reducir la diferencia entre unos objetivos ambiciosos y su aplicación práctica”, Informe Especial $n^{\circ} 31$. Luxemburgo: Tribunal de Cuentas Europeo.

Wesenberg, S., Mueller, C., Nestmann, F., Holthoff-Detto, V. (2012). "Effects of an animalassisted intervention on social behaviour, emotions, and behavioural and psychological symptoms in nursing home residents with dementia", American Journal of Alzheimer's Disease and other Dementias, 27: 8, 625-632. (Disponible online: https://doi.org/10.1177/1533317512464117).

Yánez, M. (2015). Integridad física del animal doméstico y su incidencia en los derechos constitucionales de la naturaleza. Quevedo: Universidad Técnica Estatal de Quevedo.

\section{Normas jurídicas}

Constitución de la República del Ecuador, Registro Oficial n 449, Quito, Ecuador, 20 de octubre de 2008.

Constitución Política del Estado Plurinacional de Bolivia, Gaceta Oficial, La Paz, Bolivia, 7 de febrero de 2009.

Constitución Política de los Estados Unidos Mexicanos, Diario Oficial de la Federación, Ciudad de México, México, 5 de febrero de 1917.

Ley 17/2015, de 9 de julio, del Sistema Nacional de Protección Civil, Boletín Oficial del Estado ${ }^{\circ}$ 164, Madrid, España, de 10 de julio de 2015.

Ley 45/2015, de 14 de octubre, de Voluntariado, Boletín Oficial del Estado nº 247, Madrid, España, 15 de octubre de 2015.

Ley ${ }^{\circ}$ 18.471, de Tenencia Responsable de Animales, Diario Oficial $\mathrm{n}^{\circ}$ 27714, Uruguay, República Oriental del Uruguay, 21 de abril de 2009.

Ley 11/2019, de 3 de abril, de acceso al entorno de las personas usuarias de perro de asistencia en la Comunidad de Castilla y León, Boletín Oficial del Estado n ${ }^{\circ}$ 107, Madrid, España, 4 de mayo de 2019.

Ley n 330 de Protección y Promoción del Bienestar de Animales, Diario Oficial n 82, San Salvador, El Salvador, 4 de mayo de 2016.

Ley $\mathrm{n}^{\mathrm{o}} 747$ para la Protección y Bienestar de los Animales Domésticos y Silvestres Domesticados, Diario Oficial de Nicaragua $\mathrm{n}^{\circ}$ 96, Managua, Nicaragua, 26 de mayo de 2011.

Estudios de la Paz y el Conflicto, Revista Latinoamericana, IUDPAS-UNAH, Volumen 1, Número 1, enero-junio 2020, pp. 39-58. DOI: $10.5377 /$ rlpc.v1i1.9515 
Ley n 7451 de 2017, de Bienestar de los Animales, La Gaceta n 120, San José, Costa Rica, 26 de junio del 2017.

Ley de protección de animales domésticos, Gaceta nº 70, Ciudad de Panamá, República de Panamá, 12 de octubre de 2012.

Tratado de Funcionamiento de la Unión Europea (publicado en el Diario Oficial n ${ }^{\circ} \mathrm{C} 326$ de 26 de octubre de 2012).

\section{REFERENCIA DE LOS AUTORES}

Marta Méndez Juez, PhD en Ciencia Política y de la Administración. Premio Extraordinario de Licenciatura y de Doctorado. Máster en Liderazgo Político y Dirección Pública de Instituciones Político-Administrativas y Máster en Diseño, Gestión y Evaluación de Proyectos y de Políticas Públicas por la Universidad de Alicante. Docente e investigadora en el Área de Ciencia Política y de la Administración de la Universidad de Burgos. Investigadora del Instituto de Administración Pública y del Centro de Buena Administración de la Junta de Castilla y León. Investigadora posdoctoral en el Instituto Universitario de Democracia, Paz y Seguridad de la Universidad Nacional Autónoma de Honduras y en el Servicio de Investigación Parlamentaria del Parlamento Europeo. mmjuez@ubu.es, martamendez1985@gmail.com. ORCID: 0000-0002-7670-3795

Juan Luis Castellví Guimerá, Oficial de Seguridad y Prevención del Parlamento Europeo en Bruselas. Formador interno. Vicepresidente de la Asociación Europea de Policías Sanitarios (EUPOLSAN). Gestor especialista en Seguridad y Emergencias. Perito Judicial en Adiestramiento y Conducta Canina. Experto profesional en aprendizaje animal y métodos de educación y adiestramiento orientado a perros detectores, de seguridad y rescate (UNED-España). Adiestrador Canino en Obediencia (ULPGC-España). Instructor de guías caninos para seguridad privada (Ministerio del Interior del Gobierno de España). Animal Behaviour and Welfare (The University of Edinburgh - Coursera - Reino Unido). Pet First Aid Instructor (ECSI-USA). Técnico en modificación de conducta canina y adiestrador canino profesional (ANACP juanluis.decastellvi@europarl.europa.eu, jlcastellvi@gmail.com. ORCID: 0000-0002$3049-4828$

Recibido: 01-10-2019

Aceptado: 16-10-2019

(c) (1) Licencia Creative Commons Reconocimiento 4.0 (CC BY 4.0).

Estudios de la Paz y el Conflicto, Revista Latinoamericana, IUDPAS-UNAH, Volumen 1, Número 1, enero-junio 2020, pp. 39-58. DOI: $10.5377 /$ rlpc.v1i1.9515 